\title{
Compact higher-order mode converter based on all-fiber phase plate segment
}

\author{
Y. Jung, S. U. Alam, D. J. Richardson \\ Optoelectronics Research Centre, University of Southampton, Southampton, SO17 1BJ, UK \\ ymi@orc.soton.ac.uk
}

\begin{abstract}
We propose a simple design of $L P_{11}$ spatial mode converter by splicing an all-fiber binary phase plate segment between SMF and FMF. More than $13 d B$ modal extinction ratio was successfully achieved with $4.5 \mathrm{~dB}$ insertion loss over a wide wavelength range.
\end{abstract}

\section{Introduction}

Space Division Multiplexing (SDM) technology [1-2] has been proposed and is being intensively investigated as a means to cope with our rapidly increasing data capacity requirements. In a fewmode fiber based SDM transmission system, selective mode excitation and detection is one of the most important issues and several spatial mode conversion techniques have been reported allowing the clean excitation of higherorder modes. Technical approaches demonstrated include long period fiber gratings, photonic lanterns, phase plates, spatial light modulators, fused fiber couplers and multi-plane light conversion [3, 4]. Amongst these, mode excitation using free-space phase plates has been particularly widely used in SDM transmission due to its simplicity and the high modal purities (>20dB modal extinction ratios) that can be relatively routinely achieved [2, 4]). However it has generally been implemented only with the aid of numerous free-space optical components. These are not only bulky but also expensive and subject to drifts in alignment over time. From this perspective an all-fiber approach would inevitably be much preferred and the fabrication of fully fiberized components is a prerequisite to realizing practical SDM systems.

In this paper, we propose a new type of allfiber mode converter formed simply by splicing a short segment of fiber acting as a phase plate between single-mode fiber (SMF) and few-mode fiber (FMF). As a simple example, we demonstrate an $\mathrm{LP}_{11}$ mode converter obtained by fusion splicing a commercial step-index multimode fiber (MMF) between SMF and a twomode fiber (TMF) with a large lateral offset. The core/cladding boundary of the MMF acts as an effective half-sector phase plate, providing a net $\pi$ phase shift. Detailed device design, fabrication and performance are investigated in terms of modal extinction ratio and coupling efficiency.

Device configuration and simulation results

Fig. 1(a) shows a general configuration of a free-space phase plate based mode converter. The Gaussian-like fundamental mode from an SMF is converted into the first higher-order mode $\left(\mathrm{LP}_{11}\right)$ using a binary phase plate and a pair of collimating and focusing lenses. Half of the collimated beam experiences a $\pi$-phase shift from the phase plate and this selectively excites the $\mathrm{LP}_{11}$ mode in the TMF due to the modal phase match. The phase contrast of a phase plate is generally achieved through modification of the optical path length and precise control of both the plate thickness and lateral alignment are very important in achieving high quality mode conversion.

In order to realize an all-fiber mode converter, we can consider a specialty fiber formed from 2 semi-circles of glass with different refractive indices, as shown in Fig. 1(b). Incident light splits into two optical pathways within each semi-circular section and an effective $\pi$-phase difference can be created by accumulating an optical path differences at the interface. The required length of fiber segment is less than $100 \mu \mathrm{m}$ and efficient mode coupling into the $\mathrm{LP}_{11}$ mode can be achieved without the need for any collimating/focusing lens element. To illustrate and validate the principle of operation, we used commercial step-index MMF rather than fabricating a specialty fiber.
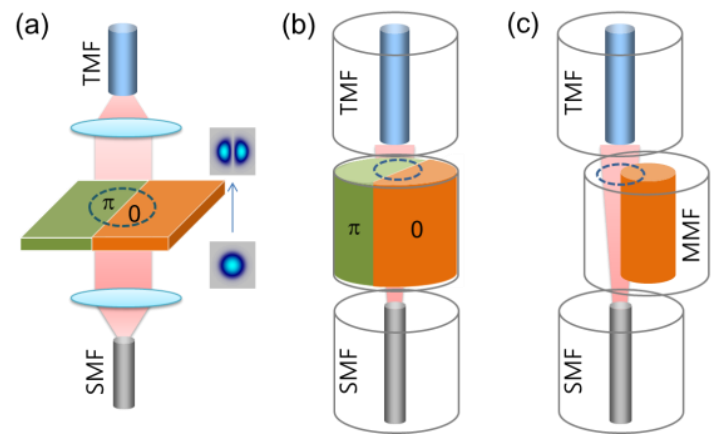

Fig. 1: Schematic diagram of (a) free-space phase plate based mode converter and all-fiber mode converter using a short fiber segment: (b) two halfcircle fiber or (b) commercial step-index MMF. 
As shown in Fig. 1(c), the MMF is fusion spliced with a large lateral offset and the core/cladding boundary of the MMF is used as an effective half-sector phase plate due to its relatively large core diameter $(50 \mu \mathrm{m})$ and large refractive index difference between the core and the cladding $(\Delta \mathrm{n}=0.016)$.
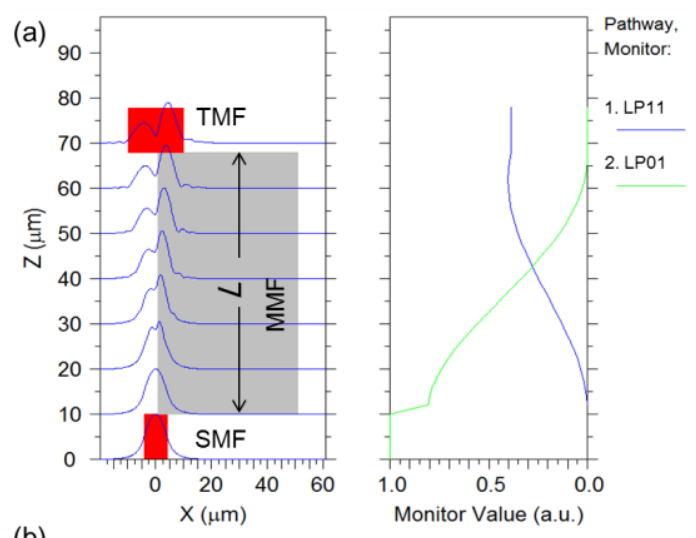

(b)

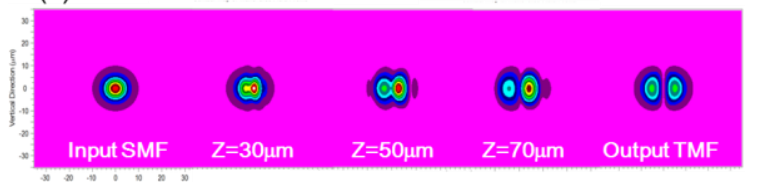

Fig. 2: (a) BPM simulation of the proposed device and calculated coupling efficiency. (b) Near-field images obtained at different positions of the MMF along the optical pathway.

To simulate wave propagation in the proposed fiber structure we performed beam propagation method (BPM) simulations using the commercial RSoft package. A short segment $(\mathrm{L}=58 \mu \mathrm{m})$ of step-index MMF was placed between the input SMF and the output TMF with a large lateral offset $(25.7 \mu \mathrm{m})$. As shown in Fig. 2(a), half of the light propagates along the core area while the other half propagates within the cladding. The input $L P_{01}$ mode from the SMF gradually converts into the $L P_{11}$ spatial mode due to the effective $\pi$-phase difference established between the two halves in the short segment of MMF. As depicted on the right side of Fig. 2(a), the monitored optical power of the $\mathrm{LP}_{11}$ mode (i.e. coupled power into the $\mathrm{LP}_{11}$ mode at the output of the TMF) is gradually increased at the expense of $\mathrm{LP}_{01}$ mode coupling efficiency. The corresponding spatial mode field evolution is visualized in Fig. 2(b). The Gaussian-like $\mathrm{LP}_{01}$ mode gradually transforms into the two-lobe intensity profile of the $\mathrm{LP}_{11}$ mode owing to the step change in refractive index at the core/cladding boundary showing that the section of MMF can be made to act like a binary phase plate.
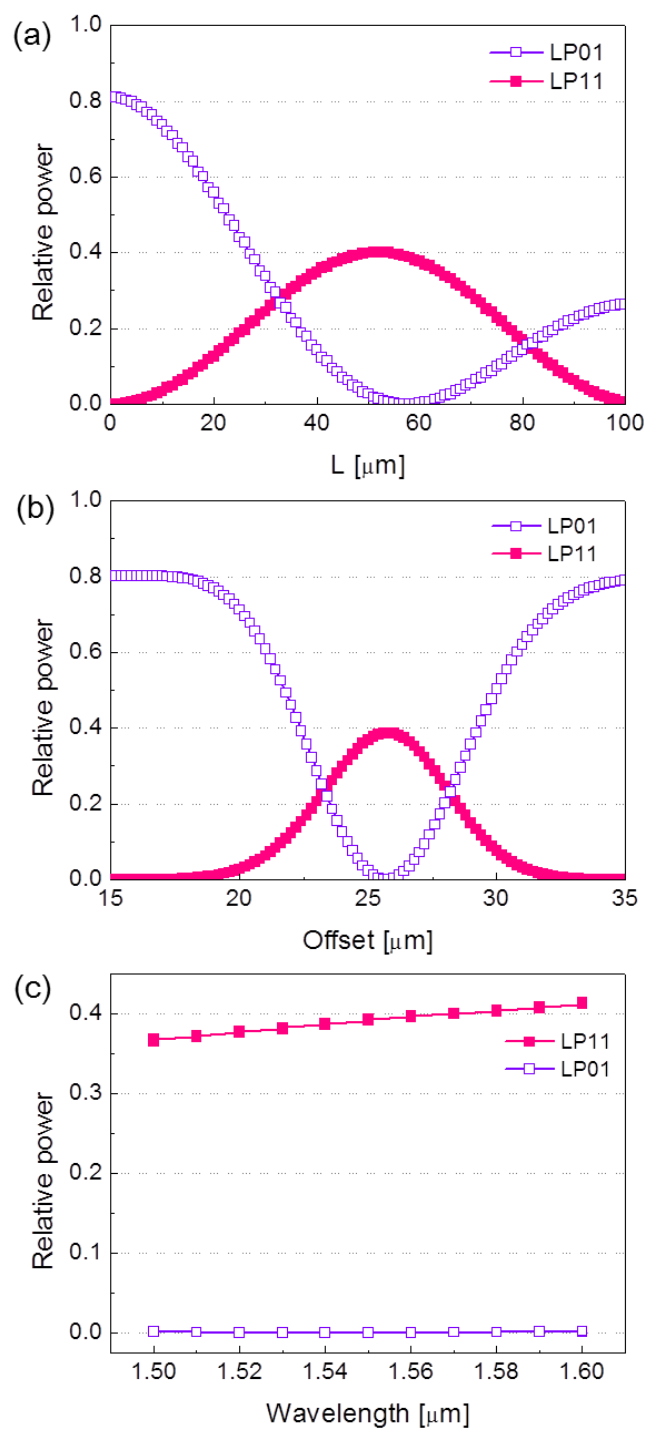

Fig. 3: The dependence of the mode coupling efficiency on (a) the length of MMF and (b) core lateral offset at $1550 \mathrm{~nm}$. (c) Operating wavelength dependence at the optimized parameters.

Fig. 3(a, b) shows the dependence of the mode coupling efficiency on the length of the MMF segment and the lateral core offset. For the optimum parameters $(L=58 \mu \mathrm{m}$, offset $=25.7 \mu \mathrm{m})$, the proposed device performs as an $\mathrm{LP}_{11}$ mode converter with high modal purity $(>30 \mathrm{~dB})$ and good $\mathrm{LP}_{11}$ mode coupling $(\sim 38 \%)$ at $1550 \mathrm{~nm}$. Any error in segment length or lateral offset will decrease the modal purity of the mode converter and therefore precise control of the segment length $(<10 \mu \mathrm{m})$ and lateral offset $(<1 \mu \mathrm{m})$ are required to get better than $20 \mathrm{~dB}$ modal extinction ratio. Fig. 3(c) shows the wavelength dependence of the mode converter at optimized parameters $(L=57 \mu \mathrm{m}$, offset $=25.7 \mu \mathrm{m})$. It shows broadband mode conversion providing better than $30 \mathrm{~dB}$ mode extinction ratio while 
maintaining more than $37 \%$ coupling efficiency over a wavelength range from $1500 \mathrm{~nm}$ to $1600 \mathrm{~nm}$. The $\mathrm{LP}_{11}$ mode coupling efficiency can be further increased to $48 \%$ by optimizing the optical mode field diameter (MFD) of the TMF.

(a)
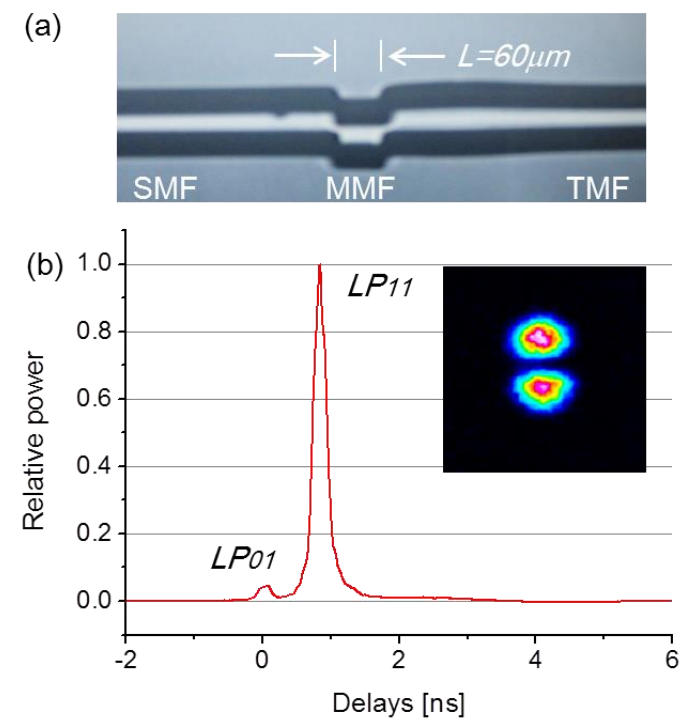

Fig. 4: Microscope image of the SMF-MMF-TMF structure and time-of-flight measurement of the proposed mode converter. Inset is the modal image after TMF at $1550 \mathrm{~nm}$.

In order to fabricate the proposed device, a length of SMF is flat cleaved and fusion-spliced to a section of MMF with a large lateral offset. The optical coupled power into the MMF was continuously monitored as a function of the offset and was stopped at $50 \%$ coupling efficiency, which means equal power within the two spatial optical pathways (i.e. core and cladding of the MMF). The MMF is then cleaved at $\sim 60 \mu \mathrm{m}$ from the SMF to MMF splice point using a micro-translation stage and a cleaver [5, 6]. The free end of the MMF is then spliced to the TMF with another large lateral offset. A Time-of-flight (ToF) measurement at $1550 \mathrm{~nm}$ was used to monitor the impulse response after $10 \mathrm{~km}$ TMF in real time to maximize the mode extinction ratio whilst performing the second offset splice. The microscope image of the fabricated all-fiber mode converter is shown in Fig. 4(a). We can clearly see that the MMF in the middle was fusion spliced with large lateral offsets between the SMF and TMF fibers with an MMF segment length of $\sim 60 \mu \mathrm{m}$. The orientation of the lateral offset planes of the two splice points should be the same for effective $L_{11}$ mode excitation and a PM fusion splicer was used to provide accurate rotational alignment. Also note that a high fusion arc power can potentially deform the splice geometry, leading for example to axial tilt. In order to avoid such deformation, cold splice conditions (i.e. low arc power and short arc duration) were used to maintain the geometry and to minimize the dopant diffusion. The two peaks in the ToF trace shown in Fig. 4(b) correspond to the $\mathrm{LP}_{01}$ and $\mathrm{LP}_{11}$ modes and their amplitudes represent the relative optical power in each of the excited modes. It is clear that clean $\mathrm{LP}_{11}$ mode excitation was achieved with a high extinction ratio $(\sim 13.6 \mathrm{~dB})$. The far-field pattern at the output of the TMF was imaged with a CCD camera to verify the dominant guided mode within the TMF. As shown in the inset of Fig. 4(b), a clean $\mathrm{LP}_{11}$ spatial mode was observed after $10 \mathrm{~km}$ length of TMF. The total insertion loss of this first demonstration device was relatively high $(\sim 4.5 \mathrm{~dB})$ however we expect that this loss can be significantly improved by optimizing the MFD of the TMF.

\section{Conclusions}

We have successfully demonstrated a simple and easy-to-fabricate $\mathrm{LP}_{11}$ spatial mode converter using an all-fiber SMF-MMF-TMF structure. The core/cladding boundary of the MMF acts like an effective binary phase plate and a $13 \mathrm{~dB}$ modal extinction ratio was achieved with $4.5 \mathrm{~dB}$ insertion loss over the entire C-band. The proposed mode converter is implemented simply by fusion splicing a few standard communication fibers together. This technique can be further extended to even higher-order mode conversion by more sophisticated engineering of the cross-section of the fiber (e.g. a four quarter-circle fiber for $\mathrm{LP}_{21}$ excitation, a circular small-core fiber for $\mathrm{LP}_{02}$ etc.).

\section{Acknowlegements}

This work was supported by the UK EPSRC grant EP/J008591/1 (COMIMO).

\section{References}

[1] D. J. Richardson et al., Nat. Photon. 7, 354 (2013).

[2] V.A.J.M. Sleiffer et al., Opt. Exp. 20, B428 (2012).

[3] Roland Ryf et al., J. Lightwave Technol. 30, 521 (2012).

[4] R. Ismaeel et al., Opt. Exp. 22, 11610 (2014).

[5] Y. Jung et al., Opt. Lett. 33, 2934 (2008).

[6] Y. Jung et al., OFC'15, paper W2A.13 (2015). 